\title{
Bewusst sprachbewusst: durch einfache Methoden der Sprach- bildung sprachliche Hindernisse im GW Schulbuch bewältigen
}

\author{
"johanna.anich@stud.sbg.ac.at, Universität Salzburg \\ **sandra.stieger@phsalzburg.at, Institut für Gesellschaftliches Lernen und Politische Bildung, PH Salzburg Stefan Zweig, ( $\triangle$ korresp. Autorin)
}

eingereicht am: 23.12.2020, akzeptiert am: 16.02.2021

Das Praxisbeispiel soll Anregungen zur Integration sprachförderlicher Methoden in den GW-Unterricht und deren Reflexion bieten. Dabei wird die Bedeutung von Sprache im Kontext fachlichen Lernens betont. In diesem Zusammenhang wird ein sprachförderliches Methodenrepertoire skizziert, das fachliches und sprachliches Lernen mit der Nutzung von GWSchulbüchern verbindet. Darüber hinaus wird ein Reflexionstool für Schüler*innen, die sogenannte „Sprachliche Werkzeugkiste“, zur sprachlichen Förderung im GW-Unterricht vorgestellt.

Keywords: Sprachliche Bildung, Sprachförderung, Geographie und Wirtschaftskunde Schulbuch, Sprachförderkonzepte, Sprachenportfolio

\section{Rising language awareness: overcome language barriers in geography and economics textbooks by using simple methods of language learning}

The article offers suggestions for integrating language-promoting methods into geography and economics (GW) education. In doing so, the importance of language in the context of subject-related learning is emphasized. In this context, a languagepromoting method repertoire is outlined, which is intended to harmonize subject-related and language learning in connection with the use of GW textbooks. Furthermore, a reflection tool for pupils, the so-called "Sprachliche Werkzeugkiste", for language support is presented.

Keywords: language education, promotion of language learning, geography and economics textbook, language promotion concepts, language portfolio

\section{$1 \quad$ Einleitung}

Aufgrund der steigenden sprachlichen Heterogenität in der Schule stellt die Unterrichtsprache Deutsch für immer mehr Schüler*innen eine Hürde dar. Dadurch kann auch der Zugang zu fachlichem Lernen verhindert oder erschwert werden (vgl. Beese et al. 2017: 4). Demzufolge ist Sprachsensibilität auch ein Anliegen für den GW-Unterricht (vgl. Budke et al. 2017: 5). Allerdings bedarf es dafür eines Bewusstseins, dass fachliches und sprachliches Lernen nicht voneinander getrennt werden können. Denn Sprache ist einerseits das zentrale Medium zur Vermittlung fachlicher Inhalte, anderseits zielt Fachunterricht auch darauf ab, im Fachkontext eine sprachliche Handlungsfähigkeit zu erwerben (vgl. Butler \& Goschler 2019: 5). So steht der bewusste Umgang mit sprachlichen Aspekten im Zentrum des sprachbewussten Fachunterrichts (vgl. Budke \& Kuckuck 2017: 7).
Zur Umsetzung eines sprachbewussten GW-Unterrichts bedarf es einer Sensibilisierung bei Lehrpersonen für potenzielle sprachliche Anforderungen heterogener Lerngruppen. Eine solche Sensibilisierung bedeutet die unterschiedlichen sprachlichen Fähigkeiten und Voraussetzungen der Lernenden bewusst wahrzunehmen und zu berücksichtigen (vgl. Budke \& Weiss 2014: 127). Eine Möglichkeit bietet der gezielte Einsatz sprachlicher Hilfen im Unterricht, um Schüler*innen sowohl zu fördern als auch zu fordern. Diese Unterrichtshilfen können sich auf verschiedene sprachliche Stolpersteine beziehen (vgl. Beese et al. 2017: 4). Vor allem Texte, wie Schulbuchtexte, können ein sprachliches Hindernis sein. Für eine systematische Erfassung derartiger potenzieller sprachlicher Herausforderungen ist eine Strukturierung auf drei Ebenen sinnvoll: der Wortebene, der Satzebene und der Textebene (vgl. Schroeter-Brauss et al. 2018: 145). 
Für den Geographieunterricht definieren Morawski und Budke (2017: 31) sowie Budke und Kuckuck (2018: $24 \mathrm{f}$.) diese drei Ebenen, die wir für das Unterrichtsfach GW leicht adaptiert haben, wie folgt:

- Die Wortebene bezieht sich auf die Nutzung geographischer und wirtschaftlicher Fachbegriffe.

- Die Satzebene erfordert ein Verständnis und die Produktion kommunikativer Sprachhandlungen. Sätze bestehen häufig aus komplexen Satzstrukturen, wie etwa Konditional- oder Relativsätzen.

- Die Textebene adressiert die geographische und wirtschaftliche Diskursfähigkeit der Lernenden. Auf dieser Ebene soll das Verständnis der Schüler*innen für fachspezifische Textmuster und ihre Produktion geschult werden. Damit verbunden ist auch die Förderung eines produktiven Umgangs mit (Geo)Medien sowie des Verständnisses für geographische und wirtschaftliche Themengebiete.

Bei der Wortebene gilt es zu berücksichtigen, dass das Verstehen von scheinbar einfachen Begriffen wie „Raum“ ein Verständnis von bedeutsamen fachlichen Konzepten erfordern kann und somit auch im Zusammenhang mit einem konzeptuellen Verständniserwerb steht. Derartige fachliche Konzepte können Lernenden „Zugänge in komplexe disziplinspezifische Denkweisen eröffnen" (Bergmeister 2017: 16), ihr Verständnis ist jedoch voraussetzungsvoll und bedarf einer Förderung kumulativer Lernprozesse (vgl. ebd.: 21).

Sprachliche Fördermaßen können diesen Konzepterwerb zusätzlich unterstützen, wenn sie nicht nur punktuell, sondern wie im vorliegenden Beitrag vorgeschlagen wird, begleitend eingesetzt werden, um auch metakognitive Lernprozesse zu stimulieren. Um sprachliche Anforderungen selbstständig zu erwerben, bedarf es jedoch an Anleitung durch die Lehrperson und Training im Unterricht (vgl. Schroeter-Brauss et al. 2018: 7). Das Praxisbeispiel möchte dazu einen Beitrag leisten und konkrete Anregungen zur Umsetzung im GW-Unterricht bieten.

\section{Didaktischeund inhaltliche Überlegungen}

„Sprache im Unterricht ist wie ein Werkzeug, das man gebraucht, während man es noch schmiedet"(Butzkamm 1989: 110).

Das Zitat von Wolfgang Butzkamm bringt den Grundgedanken des sprachbewussten Unterrichts auf den Punkt: Sprache wird in allen Unterrichtsfächern gebraucht und sprachliche Förderung ist keine alleinige Aufgabe der Sprachfächer wie Deutsch, sondern ein Anliegen für alle Unterrichtsgegenstän- de. Durch gezielte Strategien und den bewussten Einsatz verschiedener sprachförderlicher Methoden kann ein wichtiger Beitrag dazu geleistet werden, dass alle Schüler*innen Fertigkeiten der Fach- und Bildungssprache nachhaltig erwerben (vgl. Carnevale \& Wojnesitz 2014: 5). Unser Interesse liegt darin, ein sprachförderliches Methodenrepertoire für Lehrpersonen und Lernende vorzustellen, das flexibel und ohne hohen Mehraufwand und Zeitressourcen im (GW-) Unterricht eingesetzt werden kann.

Der Lehrplanbezug in der Unterstufe des sprachlichen Kompetenzerwerbs ist dabei vor allem in Bezug auf den Bildungsbereich „Sprache und Kommunikation" für das Unterrichtsfach GW gegeben (BMUKK 2000: 1044). Des Weiteren zielen die überfachlichen Unterrichtsprinzipien und Bildungsanliegen „Lesekompetenz" und "Sprachliche Bildung" auf die Sprachförderung und den Erwerb der Bildungssprache in allen Unterrichtsfächern ab (vgl. Weiglhofer 2013: 1). Auch der Grundsatzerlass zur Leseerziehung von 2017 adressiert die Bedeutung der Sprachförderung für alle Unterrichtsgegenstände (BMBWF 2017). All diese gesetzlichen Maßnahmen und Vorgaben unterstreichen die Bedeutung eines sprachsensiblen Unterrichtszugangs.

Der Beitrag soll deshalb zum einen aufzeigen, was unter sprachförderlichen Methoden und Strategien konkret im GW-Unterricht verstanden werden kann. Zum anderen soll beispielhaft am Medium Schulbuch gezeigt werden, wie derartige sprachliche Hilfen in den GW-Unterricht einfließen können. Das GWSchulbuch ist nach wie vor eines der am häufigsten genutzten Unterrichtsmedien und wird insbesondere für eigenverantwortliches Arbeiten im GW-Unterricht eingesetzt (vgl. Hintermann et al. 2014: 81). Schulbücher sollten nicht nur fachliche Lern- und Bildungsprozesse begleiten, sondern Schüler*innen auch im Spracherwerb unterstützen (vgl. BMUKK 2012: 3). Die Ergebnisse einer von uns durchgeführten exemplarischen Schulbuchanalyse zeigen, dass sich auf allen drei Ebenen sprachlicher Anforderung potenzielle sprachliche Schwierigkeiten identifizieren lassen (vgl. fachdidaktischer Beitrag im Band Anich \& Stieger). An diese Erkenntnisse knüpft das Praxisbeispiel an und stellt eine Auswahl an sprachförderlichen Methoden vor, die gemeinsam mit dem Schulbucheinsatz Anwendung finden und dadurch den identifizierten sprachlichen Hürden entgegenwirken können.

Wir zeigen beispielhaft anhand eines konkreten, zufällig ausgewählten Schulbuchauszuges aus einem GW-Lehrbuch der 1. Klasse (5. Schulstufe) auf, wie sich potenzielle sprachliche Schwierigkeiten auf Wort-, Satz- und Textebene äußern können, um die Sensibilität bei Pädagog*innen zu schärfen. Außerdem stellen wir fünf sprachförderliche Methoden vor, die 
illustrieren sollen, wie eine Kombination aus sprachlichem und fachlichem Lernen im GW-Unterricht auf allen drei Ebenen (Wort-, Satz- und Textebene) gelingen kann. Neben den ausgewählten Methoden präsentieren wir am Ende des Beitrags ein lernbegleitendes Reflexionstool, die sogenannte „Sprachliche Werkzeugkiste“. Dieses Tool soll Schüler*innen unterstützen, ihr sprachliches Wissen und Können kontinuierlich zu reflektieren und eine Sammlung an sprachlichen (Hilfs-)Methoden anzulegen, um jeder Zeit und auch fachunabhängig darauf zurückzugreifen zu können. So entsteht ein individuelles Sprachportfolio, das unterschiedliche sprachliche Werkzeuge für den (GW-)Unterricht umfasst.

\section{Sensibilisierung, Methodenauswahl und praktische Umsetzungsideen}

Die Ergebnisse der exemplarischen Schulbuchanalyse zeigen, dass potenzielle sprachliche Schwierigkeiten am häufigsten auf der Wort- und Satzebene identifizierbar sind. Anhand des folgenden Textausschnittes aus dem Schulbuch GEOprofi (Mayrhofer et al. 2019) veranschaulichen wir, wie sich mögliche sprachliche Schwierigkeiten auf Wort- und Satzebene erkennen lassen.

Die potenziellen sprachlichen Schwierigkeiten auf Wortebene sind im Textausschnitt fett hervorgehoben. Diese Analyseeinheit umfasst (komplexe) Komposita, Fremdwörter und Fachwörter, die nicht präzise und verständlich erklärt werden, sowie ambige Wörter bzw. Wortverwendungen und Nominalisierungen (vgl. BMUKK 2012: $12 \mathrm{ff}$.). Die potenziellen sprachlichen Schwierigkeiten auf Satzebene sind gestrichelt unterstrichen und kursiv gesetzt. Dazu zählen (komplexe) hypotaktische ${ }^{1}$ und parataktische ${ }^{2}$ Satzstrukturen, Passivkonstruktionen und unvollständige Sätze (BMUKK 2012: $13 \mathrm{ff}$.). In eckiger Klammer haben wir jeweils angegeben, welcher Untergruppe die Analyseeinheiten zuzuordnen sind.

\footnotetext{
„Bei einer Hypotaxe (Satzgefüge) ist ein Teilsatz dem anderen untergeordnet (subordiniert)" (Pittner \& Berman 2015: 96). Beispiel: „So verwundert es kaum, dass die riesige Insel nur ca. 60000 Einwohner zählt".

2 „Bei einer Parataxe (Satzreihung) werden strukturell gleichrangige Sätze verbunden. Dabei kann es sich um Hauptsätze handeln, aber auch um Nebensätze" (Pittner \& Berman 2015: 96). - Beispiel: „Der tiefste See der Erde liegt in Zentralasien und ist ein Süßwassersee" S. 12 in GEOprofi 1 (Mayrhofer et al. 2019).
}

Wir wollen dir nun einige Grundbegriffe [Kompositum] erklären, die du im GeografieUnterricht immer wieder brauchen wirst [...]. [Relativsatz]

Die Erde hat einen Nord- und einen Südpol [Fachwörter], die gedachte Verbindung der beiden Pole [ambiges Wort] bezeichnet man als Erdachse [Kompositum] [Relativsatz]. Eine weitere wichtige Hilfslinie [Kompositum] ist der Äquator [Fachwort]. Den Äquator [Fachwort] denken wir uns als eine um die Erde herumreichende Linie, die zu den beiden Polen [ambiges Wort und Fachwortl den gleichen Abstand hat [Relativsatz]. Der Äquator [Fachwort] teilt die Erde in eine Nord- und eine Südhalbkugel [Fachwörter und Komposita]. Österreich befindet sich auf der Nordhalbkugel [Fachwort und Kompositum]. [...]

Die Form der Erde gleicht auf den ersten Blick einer Kugel. Bei genaueren Vermessungen [Nominalisierung] konnte man jedoch feststellen, dass die Entfernung vom Erdmittelpunkt_[Kompositum] zur Erdoberfläche [Kompositum] (der Erdradius) [Kompositum und Fachwort] nicht überall exakt gleich groß ist [Konsekutivsatz]. Am Äquator [Fachwort] hat der Erdradius [Fachwort und Kompositum] eine Länge von 6378 km, an den beiden Polen [ambiges. Wort_und Fachwort] ist er jedoch um ca. $21 \mathrm{~km}$ kürzer [Parataxe]. Die Erde hat also die Form einer an den Polen [ambiges Wort] leicht eingedrückten Kugel.

Textquelle: S. 6 in GEOprofi 1 (Mayrhofer et al. 2019) Herv. d. V. - ausgenommen davon sind die Wörter Nord-, Südpol, Erdachse, Nord-, Südhalbkugel und Erdradius, die im Original fett hervorgehoben sind.

Auf der dritten Ebene sprachlicher Anforderung, also der Textebene, äußern sich die potenziellen sprachlichen Schwierigkeiten durch Textkohärenz und Textkohäsion ${ }^{4}$. Erstere erfordert seitens der Schüler*innen den Inhalt nicht nur zu verstehen, sondern auch interpretieren zu können. Diese Fähigkeiten sind durch das jeweilige lexikalische und kulturelle Wissen der Lernenden beeinflusst (vgl. Dudenredaktion 2016: 1077). Potenzielle sprachliche Schwierigkeiten in Hinblick auf die Textkohäsion betreffen nicht die

Da wir davon ausgehen, dass Leser*innen aus der Schulpraxis mit den Namen der Schulbuchautorenschaft vielfach nicht das Schulbuch assoziieren werden, führen wir ab hier nur mehr den Namen des Schulbuchs an.

4 In der Linguistik sind Kohärenz, also der inhaltliche Zusammenhang von Texten und Sätzen, und Kohäsion, die explizite Verknüpfung von Sätzen in einem Text, wesentliche Charakteristika, um Texte verstehbar und als solche interpretierbar zu machen (Glück \& Rödel 2016: 342). 
Inhaltsdimension, sondern die Textoberfläche wie Textpassagen, deren Sätze ohne Kohäsionsmittel aneinandergereiht wurden (vgl. BMUKK 2012: 14). Kohäsionsmittel sind zum Beispiel satzübergreifende Konnektoren. In diese Kategorie fallen bestimmte Adverbien ${ }^{5}$, Abtönungs- bzw. Modalpartikel ${ }^{6}$, Proformen $^{7}$, bestimmte Interpunktionszeichen ${ }^{8}$, wie auch die Wiederaufnahme (Rekurrenz') von Referenzobjekten/Elementen desselben Wortverbandes (vgl. Dudenredaktion 2016: $1081 \mathrm{f}$. und 1154f.). Da der Schulbuchausschnitt sehr kohärent gestaltet ist, wollen wir nicht auf das Fehlen dieser Kohäsionsmittel, sondern auf deren Verwendung aufmerksam machen, um dafür zu sensibilisieren. Die Mittel der Textkohäsion haben wir mit Unterstreichen gekennzeichnet und auch hier wieder in eckigen Klammern die jeweilige Unterkategorie angeführt.

\footnotetext{
5 Im Zusammenhang mit der Textkohäsion werden unterschiedliche verweisende Adverbien verwendet, wie Konnektoradverbien (z. B. schließlich, außerdem, nämlich, seitdem, etc.), Pronominal- bzw. Präpositionaladverbien (z. B. dabei, darauf, worüber, damit, danach, hiermit, etc.) oder auch Textadverbien (z. B. ferner, erstens ... zweitens, einerseits ... andererseits, etc.), die einen inhaltlichen Textzusammenhang markieren (vgl. Dudenredaktion 2016: $1086 \mathrm{f}$.)

6 „Abtönungspartikeln [...] beziehen sich weniger auf den Sachverhalt selbst als auf eine bestimmte Einstellung des Schreibers dazu" (Dudenredaktion 2016: 1087). Dazu gehören z. B. eben, ja, doch (vgl. ebd.: 1087). Beispiel: Ich bin zu Fuß gegangen. Ich besitze ja kein Auto (Herv. d. V.).

7 Zu dieser Kategorie zählen Pronomina und Artikel (vgl. Dudenredaktion 2016: 1085). Beispiel: „Die mit Abstand größte Wüste der Erde ist die Sahara. Sie erstreckt sich vom Atlantischen Ozean bis zum Roten Meer [...]“ (GEOprofi 1 2019: 6; Herv. d. V.). 8 Z. B.: „Der Doppelpunkt : signalisiert, dass etwas folgt“ (Dudenredaktion 2016: 1081). Beispiel: „Du siehst: Wir müssen auf unsere Erde gut achtgeben, [...] “" (GEOprofi 1 2019: 6; Herv. d. V.). 9 „Rekurrenz (von lat. 'wiederkehren`): Dasselbe Inhaltswort oder dieselbe Wortverbindung wird wörtlich wiederholt" (Dudenredaktion 2016: 1154). Beispiel: „Für die Höhendarstellungen in physischen Karten werden immer die Farben Grün, Gelb und Braun verwendet. Grüntöne markieren Gebiete mit geringer Höhenlage, Gelbtöne Gebiete mit mittlerer Höhe und Brauntöne Gebiete mit großer Höhe“ (GEOprofi 1 2019: 18; Herv. d. V.).
}

Wir wollen dir nun einige Grundbegriffe erklären, die du im Geografie-Unterricht immer wieder brauchen wirst $[\ldots]$.

Die Erde hat einen Nord- und einen Südpol, die gedachte Verbindung der beiden Pole bezeichnet man als Erdachse. Eine weitere wichtige Hilfslinie [Rekurrenz zu "gedachte Verbindung"] ist der Äquator. Den Äquator [Rekurrenz] denken wir uns als eine um die Erde herumreichende Linie, die zu den beiden Polen den gleichen Abstand hat. Der Äquator [Rekurrenz] teilt die Erde in eine Nordund eine Südhalbkugel. Österreich befindet sich auf der Nordhalbkugel [Rekurrenz]. [...]

Die Form der Erde gleicht auf den ersten Blick einer Kugel. Bei genaueren Vermessungen konnte man jedoch [Adverb] feststellen, dass die Entfernung vom Erdmittelpunkt zur Erdoberfläche (der Erdradius) nicht überall exakt gleich groß ist. Am Äquator hat der Erdradius [Rekurrenz] eine Länge von $6378 \mathrm{~km}$, an den beiden Polen ist er jedoch um ca. $21 \mathrm{~km}$ kürzer. Die Erde hat also [Adverb] die Form einer an den Polen leicht eingedrückten Kugel.

Textquelle: S. 6 in GEOprofi 1, Herv. d. V.-ausgenommen davon sind die Wörter Nord-, Südpol, Erdachse, Erdradius, Südhalbkugel, Nordhalbkugel Herv.i. O.

Für die Bewältigung der hier illustrativ dargestellten potenziellen sprachlichen Hürden, stellen wir nun beispielhaft einige Methoden vor, die Lernende dabei unterstützen können. In der Literatur gibt es eine Vielzahl an Methoden der Sprachförderung. Wir haben eine überschaubare und exemplarische Auswahl getroffen, die einerseits eine einfache Umsetzungsmöglichkeit für Lehrpersonen in Verbindung mit der Nutzung von Schulbüchern im (GW-)Unterricht bietet und anderseits basierend auf den Untersuchungsergebnissen der exemplarischen Schulbuchstudie (vgl. fachdidaktischer Beitrag im Band Anich \& Stieger) ausgewählt wurde. Wir stellen diese Methoden kurz für die jeweiligen Ebenen vor:

\section{- Sprachliche Hindernisse auf Wortebene meistern: „Scaffolding“}

Die Methode des "Scaffoldings" dient dazu, Schüler*innen beim Erwerb der Bildungs- und Fachsprache zu unterstützen. „Scaffold“ (engl.) kann mit „(Bau-)Gerüst“ übersetzt werden. Im Kontext der Sprachbildung ist damit „die Unterstützung von Lernprozessen durch die Bereitstellung sprachlicher Hilfen durch die Lehrperson 
[...]" (Carnevale \& Wojnesitz 2014: 14) gemeint. Zur Methode zählen beispielsweise Wortlisten und Erklärungen, aber auch Bildimpulse (z. B. Verbildlichung von Begriffen). Diese Methoden des "Scaffoldings" können je nach Kompetenzstand der Schüler*innen differenziert eingesetzt und bei einem Kompetenzzuwachs stufenweise abgebaut werden (vgl. ebd.: $14 \mathrm{f}$.).

\section{- Sprachliche Hindernisse auf Wort- und Satz- ebene meistern: „Textpuzzle“}

Bei dieser Methode erhalten die Lernenden ungeordnete Satzfragmente wie einzelne Satzteile oder Wörter, die sie in sprachlich sinnvolle Einheiten bzw. in einer passenden Reihenfolge zusammensetzen sollen. Das unterstützt auch das Training des Fachwortschatzes, da Schüler*innen die einzelnen Textbausteine mehrmals lesen, um das Puzzle zu lösen können. Derartige Aufgaben können das Interesse der Schüler*innen an einem Sachtext zusätzlich wecken, da diese zu einer aktiven Auseinandersetzung angeregt werden. Außerdem lässt sich der Schwierigkeitsgrad je nach Gestaltung der „Puzzleteile“ und nach Bekanntheit des Textes variieren (vgl. Hepp et al. 2003/2005: 4).

\section{- Sprachliche Hindernisse auf Textebene meistern: „Strukturdiagramm“}

Ein Strukturdiagramm ermöglicht eine netzartige, abstrakte und visuelle Darstellung bestimmter Sachverhalte in ihrem Zusammenhang. Die Lernenden ordnen dabei wichtige Fachbegriffe in einer für sie verzweigten Struktur an, die ihre Logik und innere Struktur wiedergibt. Dadurch können verschiedene Prozesse und Handlungen visuell dargestellt und beschrieben werden. Diese Methode hilft Schüler*innen Zusammenhänge zu erkennen, darzustellen und unterstützt sie auch diese zu verbalisieren. Das Strukturdiagramm kann je nach Schwierigkeitsgrad mit den Lernenden nach dem Lesen gemeinsam, in Partnerarbeit oder selbstständig erstellt werden (vgl. Hepp et al. 2003/2005: 4).

\section{- Sprachliche Hindernisse auf Wort-, Satz- und Textebene meistern: „Verstehensinseln“}

Die Texterschließung anhand von „Verstehensinseln" beginnt mit jenen Textteilen, die die Schüler*innen schon verstehen. Die Sammlung von Textelementen, die bekannt sind, dient als positive Verstärkung der eigenen Fähigkeiten, hat also eine motivierende Wirkung. In Kombination mit verschiedenen Lesestrategien (z. B. wiederholtes Le- sen), können die Lernenden ihre „Verstehensinsel“ mit jedem Lesedurchgang ausweiten (vgl. Leisen 2015: 15; Rosebrock \& Nix 2017: 82).

\section{- Sprachliche Hindernisse auf Textebene meistern: „Fragen an den Text stellen“}

Diese sprachförderliche Methode regt Schüler*innen vor allem dazu an, sich vertieft mit Textinhalten auseinanderzusetzen und sich unbekannten Texten neugierig zu widmen. Dabei wird explizit auch das Vorwissen aktiviert, da sie in einem ersten Schritt versuchen Fragen durch Hypothesen oder Vermutungen ohne den Text zu lesen zu beantworten. In einer einfachen Version oder zum Einführen der Methoden in den Unterricht erhalten die Schüler*innen vorgegebene Fragen. In einem zweiten Schritt oder wenn die Methode schon bekannt ist, sollen die Schüler*innen selbstständig Fragen zum Text formulieren. So können Lernende Texte individuell bewusst erschließen und auf ihren Erklärungsgehalt für die für sie relevanten Fragen zu einem Thema prüfen (vgl. Oleschko et. al 2016: $200 \mathrm{f}$.).

Die konkrete Umsetzung der hier angeführten sprachförderlichen Methoden im GW-Unterricht haben wir im Anhang als Anwendungsbeispiel für die Unterrichtspraxis skizziert.

\section{Reflexionstool: „Sprachliche Werkzeug- kiste“}

Dieses Portfolio, das von den Lernenden ausgewählte Methoden umfasst, ermöglicht sowohl eine strukturierte als auch dem Prinzip der Individualisierung folgende sprachliche Förderung. Ein solches Portfolio kann die gesamte Sekundarstufe und alle Fächer einbeziehen. Die „Sprachliche Werkzeugkiste“ erlaubt es Lernenden ihre sprachlichen Methoden selbstbestimmt zu sammeln und veranschaulicht ihnen, über welches sprachliche Wissen und Können sie bereits verfügen (vgl. Leutke 2002: 2).

Um die Schüler*innen in ihrer Auswahl zu unterstützen, bedarf es zu Beginn der Sekundarstufe möglicherweise Hilfestellungen und Hinweise seitens der Lehrperson. Es sollte jedoch eine schrittweise Entwicklung hin zu einer selbstständigen und selbstbestimmten Bearbeitung der „Sprachliche Werkzeugkiste" erfolgen und den Lernenden keine Methoden aufgezwungen werden.

Dieses Sprachportfolio dient jedoch nicht nur dem Sammeln von sprachlichen Werkzeugen, sondern bietet auch Platz für die Reflexion über die eigenen 
sprachlichen Fähigkeiten. Dabei sollen alle drei Ebenen (fach-)sprachlicher Anforderung, also die Wort-, Satz-, Textebene angesprochen werden (vgl. Morawski \& Budke 2017: 31; Budke \& Kuckuck 2017: 24 f.). Hierzu können bestimmte Visualisierungstechniken, wie Mindmaps oder Strukturdiagramme, eingebunden werden. Die „Sprachliche Werkzeugkiste“ kann auch im Sinne einer "Lernfortschrittsdokumentation“ Einsatz finden, indem sie Lernerfolge sichtbar macht (vgl. Eder et al. 2009: 255).

Ein Vorschlag für konkrete „sprachliche Werkzeuge", also sprachsensible Methoden in Bezug auf den GW-Unterricht, findet sich im Anhang.

\section{Dank}

Wir danken unseren beiden anonymen Gutachter*innen für ihre konstruktiven Kommentare und Verbesserungsvorschläge sowie der Pädagogischen Hochschule Stefan Zweig für die Unterstützung dieser Publikation.

\section{Literatur}

Beese, M., A. Kleinpaß, S. Krämer, M. Reschke, S. Rzeha \& M. Wiethoff (2017): Praxishandbuch Sprachbildung Biologie: Sprachsensibel unterrichten - Sprache fördern. Klett Sprachen, Stuttgart.

Bergmeister, F. M. (2017): Schwellenkonzepte als Zugänge fachlichen Verstehens - Wege zur differenzierten Erschließung komplexer (ökonomischer) Basiskonzepte im GW-Unterricht. In: GW Unterricht 147. S. 16-25. DOI: https://doi.org/10.1553/gw-unterricht147s16

Budke, A. \& M. Kuckuck (2017): Sprache im Geographieunterricht. In: Budke, A. \& M. Kuckuck (Hrsg.): Sprache im Geographieunterricht: Bilinguale und sprachsensible Materialien und Methoden. Waxmann, Münster. S. 7-35.

Budke, A., M. Kuckuck \& M. Morawski (2017): Sprachbewusste Kartenarbeit? Beobachtungen zum Karteneinsatz im Geographieunterricht. In: GW-Unterricht 148. S. 5-15. DOI: https://doi.org/10.1553/gw-unterricht148s5

Budke, A. \& G. Weiss (2014): Sprachsensibler Geographieunterricht. In: Michalak, M. (Hg.): Sprache als Lernmedium in allen Fächern. Schneider, Baltmannsweiler. S. 113-133.

BMUKK - Bundesministerium für Unterricht, Kunst und Kultur (2012): Sprache in Schulbüchern: Empfehlungen zur Sprachverwendung in Schulbüchern für SchulbuchautorInnen, GutachterInnen und Schulbuchverlage. Digitales Druckzentrum des BMUKK, Wien.

BMUKK - Bundesministerium für Unterricht Kunst und Kultur (2000): Lehrplan der AHS-Unterstufe für Geographie und Wirtschaftskunde. Bildungs- und Lehraufgabe. In: Bundesgesetzblatt II Nr. 133 v. 11.5.2000; S. 1044-1048 https://www.ris.bka.gv.at/Dokumente/BgblPdf/2000_133_2/2000_133_2.pdf (29.03.2021)

BMBWF - Bundesministerium für Bildung, Wissenschaft und Forschung (2017): Grundsatzerlass Leseerziehung. https://www.bmbwf.gv.at/Themen/schule/schulrecht/ rs/1997-2017/2013_11.html (08.12.2020)
Butler, M. \& J. Goschler (2019): Zur Einleitung: Dimensionen der Sprachsensibilität im Fachunterricht - Perspektiven und Fragerichtungen. In: Butler, M. \& J. Goschler (Hrsg.): Sprachsensibler Fachunterricht: Chancen und Herausforderungen aus interdisziplinärer Perspektive. Springer, Wiesbaden. S. 5-13.

Butzkamm, W. (1989): Psycholinguistik des Fremdsprachenunterrichts. Natürliche Künstlichkeit: Von der Muttersprache zur Fremdsprache. Francke, Tübingen.

Carnevale, C. \& A. Wojnesitz (2014): Sprachsensibler Fachunterricht in der Sekundarstufe: Grundlagen - Methoden - Praxisbeispiele. (= ÖSZ Praxisreihe Heft 23). ÖSZ, Graz.

Dudenredaktion (2016): Die Grammatik: Unentbehrlich für richtiges Deutsch. Bibliographisches Institut $\mathrm{GmbH}$, Leipzig.

Eder, F., G. H. Neuweg \& J. Thonhauser (2009): Leistungsfeststellung und Leistungsbeurteilung. https://www.bifie. at/material/nationale- bildungsberichterstattung/nationaler-bildungsbericht-2009/ (16.11.2020)

Glück, H. \& M. Rödel (Hrsg.) (2016): Metzler Lexikon Sprache (5.Aufl.). Metzler, Stuttgart.

Hepp, R., A. Krüger \& J. Leisen (2003/2005): Steckbrief Methoden-Werkzeuge. Friedrich, Seelze.

Hintermann, C., C. Markom, H. Weinhäupl \& S. Üllen (2014): Debating Migration in Textbooks and Classrooms in Austria. In: Journal of Educational Media, Memory, and Society 6. S. 79-106. DOI: https://doi. org/10.3167/jemms.2014.060105

Leisen, J. (2015): Leseverstehen und Leseförderung als Aufgabe aller Fächer. In: Pädagogik.Leben 1. S. 14-15. http://www.josefleisen.de/downloads/lesen/10\%20 Leseverstehen $\% 20$ als $\% 20$ Aufgabe $\% 20$ aller $\% 20$ F\%C3\%A4cher\%20P\%C3\%A4dagogik-leben\%20 2015.pdf (24.02.2021)

Leutke, M. K. (2002): Mein Sprachenportfolio: Handreichungen für Lehrerinnen und Lehrer. https://c.wgr.de/d/ 94df8e4d2ad73ed29ef8070bc339beabf1 18c3970507c5 ac6bdbb10d4ca09b7a.pdf/978-3-425-02130-0-2-1.pdf (03.12.2020)

Mayrhofer, G., R. Posch \& I. Reiter (2019): GEOprofi 1: Geographie und Wirtschaftskunde für die 5. Schulstufe (2. Aufl.). Veritas, Linz. S. 6-14; S. 16-26.

Morawski, M. \& A. Budke (2017): Sprachförderkonzepte in bilingualen Geographieschulbüchern. In: GW Unterricht 148. S. 28-42. DOI: https://doi.org/10.1553/gwunterricht148s 28

Oleschko, S., B. Weinkauf \& S. Wiemers (2016): Praxishandbuch Sprachbildung Geographie: Sprachsensibel unterrichten - Sprache fördern (1. Aufl.). Klett Sprachen, Stuttgart.

Pittner, K. \& J. Berman (2015): Deutsche Syntax: Ein Arbeitsbuch (6. Aufl.). Narr, Tübingen.

Rosebrock, C. \& D. Nix (2017): Grundlagen der Lesediagnostik und der systematischen schulischen Leseförderung (8. Aufl.). Schneider, Baltmannsweiler.

Schroeter-Brauss, S., V. Wecker \& L. Henrici (2018): Sprache im naturwissenschaftlichen Unterricht: Eine Einführung. Waxmann, Münster.

Weiglhofer, H. (2013): Die Kompetenzenlandkarte für Unterrichtsprinzipien und Bildungsanliegen. https:// www.bmbwf.gv.at/Themen/schule/schulpraxis/uek.html $(03.12 .2020)$ 


\section{Arbeitsmaterial}

Der zufällig gewählte Schulbuchausschnitt aus GEOprofi 1 (Mayrhofer et al. 2019) dient als Beispiel für alle hier vorgestellten Methoden und findet in den folgenden Aufgaben immer Verwendung. Aus Platzgründen ist der Textausschnitt nur hier angeführt:

\section{M1 Textbeispiel}

Wir wollen dir nun einige Grundbegriffe erklären, die du im Geografie-Unterricht immer wieder brauchen wirst [...].

Die Erde hat einen Nord- und einen Südpol, die gedachte Verbindung der beiden Pole bezeichnet man als Erdachse. Eine weitere wichtige Hilfslinie ist der Äquator. Den Äquator denken wir uns als eine um die Erde herumreichende Linie, die zu den beiden Polen den gleichen Abstand hat.

Der Äquator teilt die Erde in eine Nord- und eine Südhalbkugel. Österreich befindet sich auf der Nordhalbkugel. [...]

Die Form der Erde gleicht auf den ersten Blick einer Kugel. Bei genaueren Vermessungen konnte man jedoch feststellen, dass die Entfernung vom Erdmittelpunkt zur Erdoberfläche (der Erdradius) nicht überall exakt gleich groß ist. Am Äquator hat der Erdradius eine Länge von 6378 km, an den beiden Polen ist er jedoch um ca. 21 km kürzer. Die Erde hat also die Form einer an den Polen leicht eingedrückten Kugel.

Textquelle: S. 6 in GEOprofi 1 (Mayrhofer et al. 2019: 6; Herv. i. O.)

\section{Scaffolding (vgl. Carnevale \& Wojnesitz 2014: 14f.)}

Das Scaffolding-Prinzip haben wir mittels einer Wortliste und Erklärungen umgesetzt. Derartige Wortlisten können vor allem für DaZ-Schüler*innen, also für Lernende mit Deutsch als Zweitsprache, als Sprachförderung eingesetzt werden und ihnen bei der Verbalisierung fachsprachlicher Inhalte helfen. Wenn möglich sollten zur Unterstützung auch der Artikel des Fachbegriffs und der Plural ergänzt werden.

Im Text verbergen sich viele wichtige Wörter (= Grundbegriffe) für den GW-Unterricht, denen du immer wieder begegnen wirst.

1a Lies dir den Text (M1) aufmerksam durch und unterstreiche im Text alle Grundbegriffe der Wortliste (M2).

1b Trage in die leere Spalte der Wortliste (M2) eine mögliche eigene Erklärung (= dein eigener Merksatz) für den Grundbegriff ein. Du kannst auch ein Bild des Grundbegriffs malen.

Die Methode lässt sich beliebig variieren, indem zum Beispiel Erklärungen für Grundbegriffe zugeordnet werden müssen oder sich ein Fehler in den Erklärungen der Grundbegriffe versteckt. Eine Möglichkeit ist auch das Sammeln von Beispielsätzen aus Medienberichten.

Für einen bewussten konzeptuellen Verständnisaufbau sollten bei bedeutsamen Begriffen weitere Spalten ergänzt werden, in denen die Lernenden neue Bedeutungen der Grundbegriffe dokumentieren können (z. B. wären die Großformen der Erdoberfläche eine mögliche Erweiterung). Dabei gilt es bei Lernenden ein Bewusstsein zu schaffen, dass sie diese Grundbegriffe benötigen, um wie hier beispielhaft gezeigt werden kann, das fachliche Konzept des Aufbaus der Erde zu verstehen (vgl. Bergmeister 2017). 
M2 Wortliste zum Text

\section{Hier findest du einige Grundbegriffe für den GW-Unterricht}

\begin{tabular}{l|c}
$\begin{array}{c}\text { Wichtiges Wort } \\
\text { (= Grundbegriff) mit Artikel und } \\
\text { in der Mehrzahl }\end{array}$ & $\begin{array}{c}\text { Meine Erklärung für den Grundbegriff } \\
\text { (du kannst auch anstelle einer Erklärung ein Bild } \\
\text { oder eine Skizze zeichnen) }\end{array}$ \\
\hline Pol, der; die Pole & $\begin{array}{l}\text { Erdachse, die } \\
\text { Äquator, der }\end{array}$ \\
\hline Nordhalbkugel, die & \\
\hline Südhalbkugel, die & \\
\hline Erdmittelpunkt, der & \\
\hline Erdoberfläche, die \\
\hline Erdradius, der
\end{tabular}

2 „Textpuzzle“ (vgl. Hepp et al. 2003/2005: 4)

2a Lies dir den Text (M1) durch.

2b Die Textteile (M3) sind in Unordnung geraten. Schaffst du es, ohne nachzulesen, die Textteile wieder in eine sinnvolle Reihenfolge zu ordnen? Schneide die Textteile aus und ordne sie in eine passende Reihenfolge.

M3 Textteile

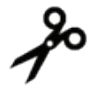

Den Äquator denken wir uns als eine, um die Erde herumreichende Linie,

die zu den beiden Polen den gleichen Abstand hat.

einer an den Polen leicht eingedrückten Kugel.

an den beiden Polen ist er jedoch um ca. 21 km kürzer.

Die Form der Erde gleicht auf den ersten Blick einer Kugel. Bei genaueren Vermessungen konnte man jedoch feststellen,

Die Erde hat also die Form Am Äquator hat der Erdradius eine Länge von 6378 km,

in eine Nord- und eine Südhalbkugel.

\begin{tabular}{|l|l|}
\hline Österreich befindet sich auf der Nordhalbkugel. & der Äquator. \\
\hline
\end{tabular}

die gedachte Verbindung der beiden Pole bezeichnet man als Erdachse.

Der Äquator teilt die Erde Eine weitere wichtige Hilfslinie ist

Die Erde hat einen Nord- und einen Südpol,

dass die Entfernung vom Erdmittelpunkł zur Erdoberfläche (der Erdradius) nicht überall exakł gleich groß ist. 
Der Methodeneinsatz des Textpuzzles ist sehr einfach. Es reicht eine Kopie einer passenden Textstelle aus dem Schulbuch, die in Textfragmente zerschnitten wird und dann als Arbeitsmaterial für die Schüler*innen dient. Da das Ausschneiden zeitintensiv ist, können Textteile natürlich auch in einer digitalisierten Form (z. B. auf PowerPoint) angeboten werden.

\section{3 „Verstehensinseln“(vgl. Leisen 2015: 15)}

3a Lies zuerst den Text (M1) aufmerksam durch.

3b Wiederhole deinen Lesevorgang.

3c Notiere dann alle wichtigen Begriffe des Textes, die du bereits verstehst, im inneren Kreis der „Verstehensinsel“ (M4). Außerdem kannst du die Begriffe, die du noch nicht verstanden hast, außerhalb deiner „Verstehensinsel“ (M4) notieren. Bei dieser Aufgabe können dir folgende Fragen helfen:

- Wie stellen wir Menschen uns die Form der Erde vor?

- Welche gedachten Linien sind für unsere Vorstellung der Erde wichtig?

3d Formuliere einen kurzen eigenen Text mit den wichtigen Begriffen.

\section{M4 Meine Verstehensinsel zum Text „Grundbegriffe für die Beschreibung der Erde”}

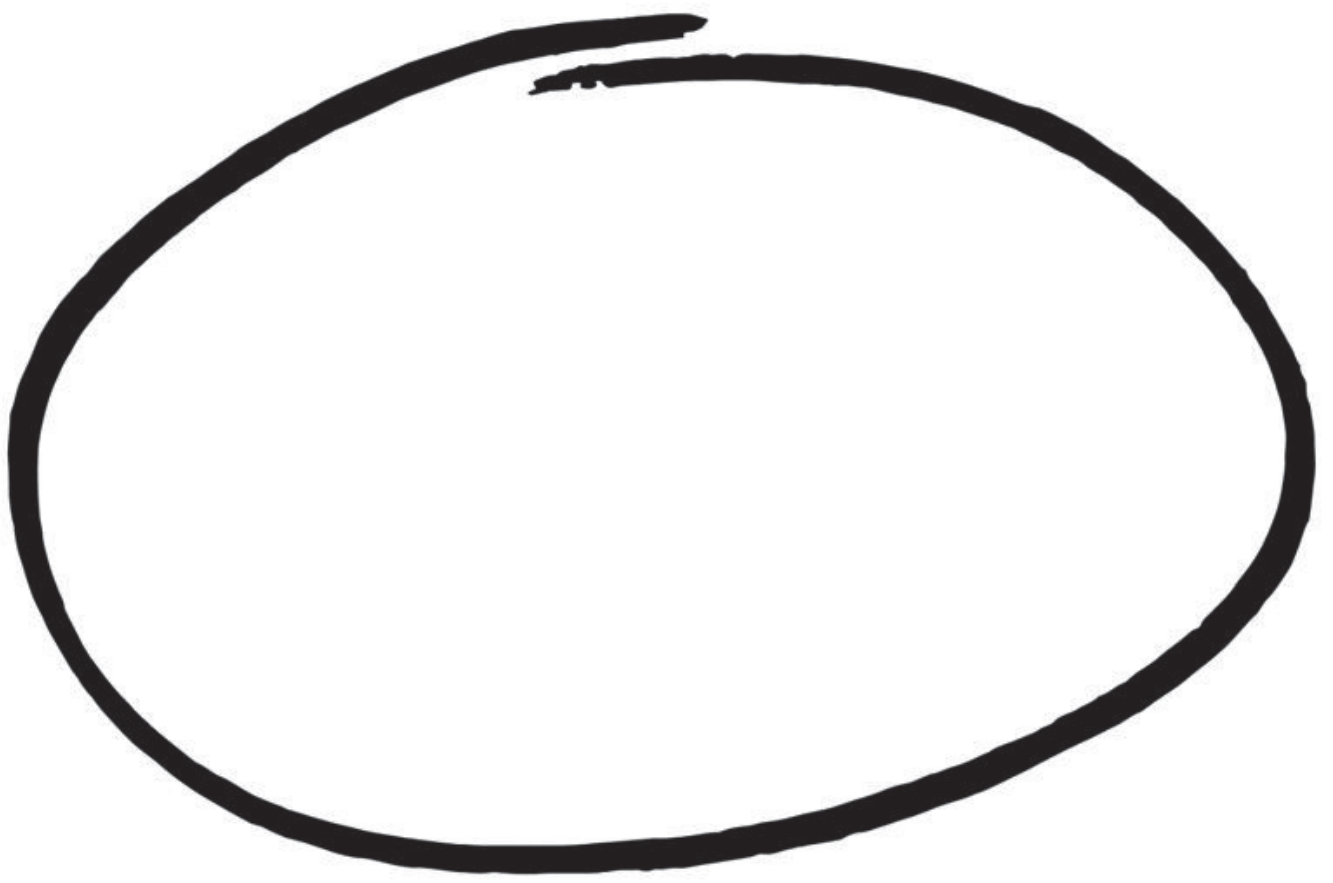

\section{Mögliche Schüler*innenlösung}

zu 3c Individuelle Ergebnisse - z. B.: Kugel, Pole, Nord- und Südpol, Erdachse, Äquator, Erdradius, Erdmittelpunkt, Erdoberfläche

zu 3d Ich stelle mir die Erde wie eine Kugel vor, die oben und unten leicht eingedrückt ist. Diese Stellen heißen Pole. Also eine Kugel, die an den Polen flacher ist. Es gibt einen Nord- und einen Südpol.

Wichtige gedachte Linien sind die Erdachse, der Äquator und der Erdradius. Die Erdachse verbindet den Nord- und Südpol. Der Äquator reicht wie ein Gürtel um die Erde herum und hat den gleichen Abstand zu den Polen. Der Erdradius gibt die Entfernung vom Erdmittelpunkt zur Erdoberfläche an. 
4 „Fragen an den Text stellen“ (vgl. Oleschko et al. 2016: 200 f.)

4a Deck zuerst die Spalte auf der rechten Seite des Textes (M5) mit einem Blatt Papier oder mit deiner Hand ab. Lies die Fragen aufmerksam durch. Kannst du die Fragen beantworten? Schreibe deine Antworten unter die Fragen.

4b Lies dann den Text (M5) in der linken Spalte. Versuche die Frage mit Hilfe des Textes zu beantworten.

$4 \mathrm{c}$ Arbeitet zu zweit. Vergleicht eure Antworten.

\section{M5 Frage und Antwort}

Die Erde hat einen Nord- und einen Südpol, die gedachte Verbindung der beiden Pole bezeichnet man als Erdachse. Eine weitere wichtige Hilfslinie ist der Äquator. Den Äquator

denken wir uns als eine um die Erde herumreichende Linie, Was ist der Äquator? die zu den beiden Polen den gleichen Abstand hat. Der Äquator teilt die Erde in eine Nord- und eine Südhalbkugel. Österreich befindet sich auf der Nordhalbkugel. [...]

Warum gibt es einen Nord- und Südpol?

Warum brauchen wir diese Begriffe?

Textquelle: S. 6 in GEOprofi 1 (Herv. i. O.)

4d Lies dir nun den Text (M6) über unsere Vorstellung der Erde durch. Bevor du den Text liest, stelle drei Fragen an den Text und notiere sie in die rechte Spalte. Lies den Text. Prüfe, ob du deine Fragen mit dem Text beantworten kannst. Schreibe die gefundenen Antworten ebenfalls in die rechte Spalte.

\section{M6 Unsere Vorstellungen der Erde}

Die Form der Erde gleicht auf den ersten Blick einer Kugel.

Bei genaueren Vermessungen konnte man jedoch feststellen, dass die Entfernung vom Erdmittelpunkt zur Erdoberfläche (der Erdradius) nicht überall exakt gleich groß ist. Am Äquator hat der Erdradius eine Länge von 6378 km, an den beiden Polen ist er jedoch um ca. $21 \mathrm{~km}$ kürzer. Die Erde hat also die Form einer an den Polen leicht eingedrückten Kugel.

Textquelle: S. 6 in GEOprofi 1 (Herv. i. O.)

\section{Mögliche Schüler*innenlösung}

$\mathrm{zu} 4 \mathrm{~b} / \mathrm{c}$

Mögliche Antworten der Schüler*innen:

- Warum gibt es einen Nord- und Südpol?

$\bigcirc$ Es gibt einen Nord- und Südpol damit wir sehen, wo der nördlichste oder südlichste Punkt der Erde ist.

Vielleicht hat das auch etwas mit Magneten zu tun, der Kompass zeigt doch immer nach Norden?

- Was ist der Äquator?

$\bigcirc$ Der Äquator ist eine Linie, die ich mir vorstelle, um die Erde in eine Nord- und eine Südhalbkugel zu teilen.

$\bigcirc$ Der Äquator ist eine gedachte Hilfslinie. Er reicht einmal um die Erde rundherum - wie ein Gürtel.

- Warum brauchen wir diese Grundbegriffe?

Wir brauchen diese Grundbegriffe, weil sie im GW-Unterricht oft verwendet werden.

$\mathrm{zu} 4 \mathrm{~d}$

$\bigcirc$ Wir brauchen diese Grundbegriffe, um die Erde zu beschreiben.

Individuelle Fragen. Mögliche Fragen können sein: Wie sieht unsere Erde aus? Kann man die Erde abmessen? Wie lang sind $6378 \mathrm{~km}$ ? Was sind Pole genau? 
5 Strukturdiagramm (vgl. Hepp et al. 2003/2005: 4).

5a Lies den Text (M1) aufmerksam durch.

$5 \mathrm{~b}$ Wiederhole deinen Lesevorgang.

5c Erstellt zu zweit mit Hilfe der Vorlage M7 ein Strukturdiagramm zu den Textinhalten zum Thema: Unsere Vorstellung der Erde.

- Versucht zuerst eine Überschrift für die Inhalte des Textes zu finden.

- Überlegt dann, in welche kleineren Themen (= Sinnabschnitte) sich der Text weiter untergliedern lässt. (Tipp: Die Hervorhebungen und Absätze können euch dabei helfen.)

- Können diese Sinnabschnitte (Themen) miteinander in Verbindung gebracht werden? Verbindet die Linien mit einem passenden Verb (= Zeitwort).

\section{M7 Vorlage Strukturdiagramm}

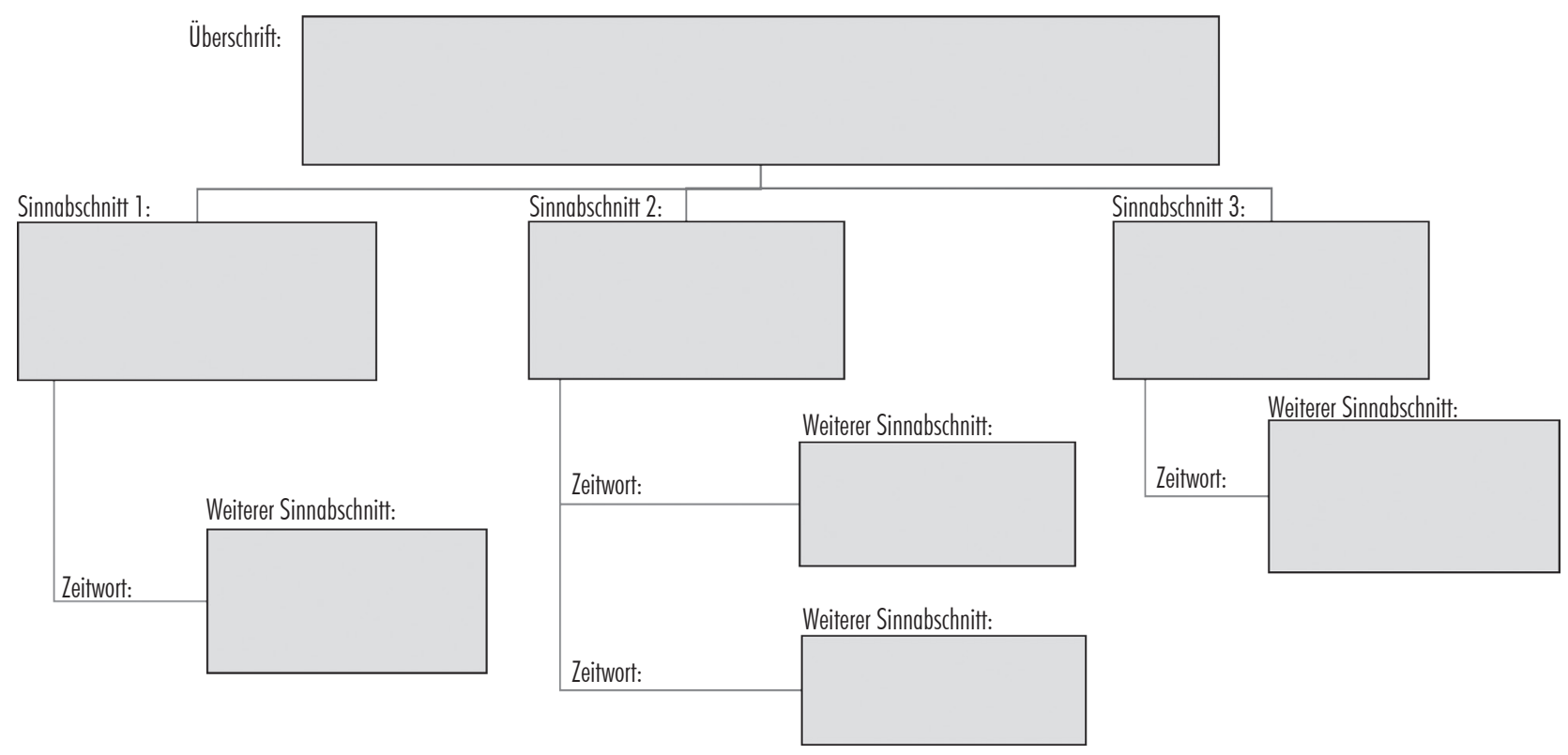

\section{Mögliche Schüler*innenlösung}

zu 5 c

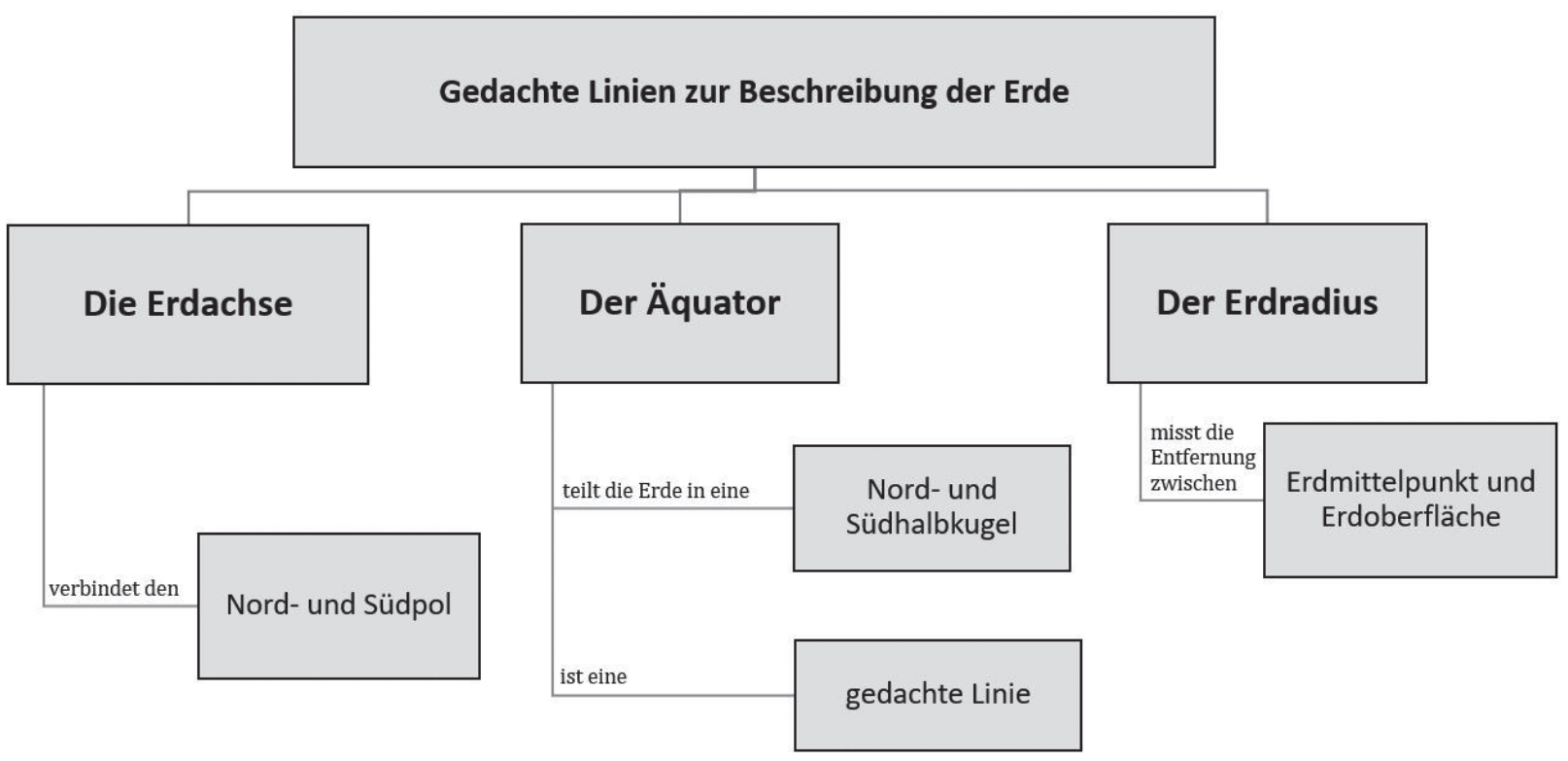



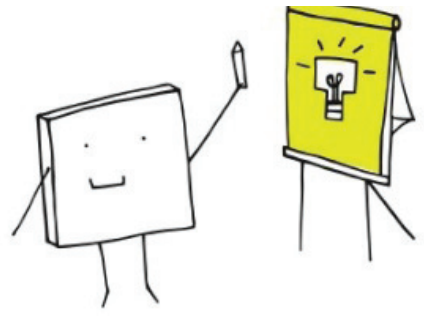

\author{
M8 Handout Methoden (für Lehrpersonen)
}

\title{
SPRACHLICHE WERKZEUGKISTE FÜR DEN (GW-)UNTERRICHT
}

\begin{abstract}
Die sprachliche Werkzeugkiste dient als Reflexionstool der sprachlichen Fähigkeiten seitens der Schüler*innen im (GW-) Unterricht. Durch das regelmäßige Sammeln von Inhalten und Überlegungen entsteht eine Art Portfolio, welches das sprachliche Wissen und Können der Schüler*innen veranschaulicht. Diese Portfolioarbeit umfasst dabei alle drei Ebenen (fach-)sprachlicher Anforderung, also die Wort-, Satz- und Textebene (vgl. Morawski \& Budke 2017: 31; vgl. Budke \& Kuckuck 2018: 24f.).

Für die konkrete Umsetzung im (GW-)Unterricht finden Sie nun einige Ideen, die beispielsweise nach jedem größeren Themenblock im Unterricht Platz finden können. Die Methoden können auch den Einsatz von Schulbüchern begleiten. Am Beginn der Sekundarstufe I können Fokusfragen oder Instruktionen bzw. Anregungen seitens der Lehrperson den Methodeneinsatz und ihre Auswahl begleiten. Durch regelmäßigen Einsatz des Tools kann und soll es aber zu einer eigenständigen Umsetzung, also Befüllung der sprachlichen Werkzeugkiste, durch die Schüler*innen kommen. Die strukturierte und individuelle Sammlung sprachlicher Werkzeuge regt zu ihrer Reflexion und vertiefenden Auseinandersetzung mit dem neu erworbenen fach- bzw. bildungssprachlichen Wissen und Können an.
\end{abstract}

\section{Wortebene:}

Diese Ebene beschäftigt sich vor allem mit dem Erwerb geographischer und wirtschaftlicher Fachbegriffe und mit der Erweiterung des (Fach-) Wortschatzes (vgl. Morawski \& Budke 2017: 31; vgl. Budke \& Kuckuck 2018: 24f.). Im Zusammenhang mit der sprachlichen Werkzeugkiste bietet es sich an, dass Schüler*innen zu den zentralen Fachbegriffen eines Themas eigene Definition festhalten. Passende eigene Zeichnungen oder eingeklebte Bilder können der Visualisierung dienen (= Methode Scaffolding). Des Weiteren können Schüler*innen einen für sie wichtigen Schlüsselbegriff zum behandelten Themengebiet bestimmen und diesen beispielsweise als „Wort der Woche“ in ihrer sprachlichen Werkzeugkiste sammeln. Hier kann auch die Methode „Themen-ABC“ Anwendung finden. Dabei notieren die Schüler*innen alle wichtigen Fachbegriffe, die ihnen zu einem bestimmen Themengebiet einfallen, in möglichst alphabethischer Reihenfolge (vgl. Carnevale \& Wojnesitz 2014: 14; vgl. Hepp, Krüger \& Leisen 2003/2005: 4).

\section{Satzebene:}

Die Satzebene behandelt das Verständnis sowie die Produktion kommunikativer Sprachhandlungen und damit auch den Erwerb (fach-) spezifischer Textstrukturen (vgl. Morawski \& Budke 2017: 31; vgl. Budke \& Kuckuck 2018: 24f.; vgl. Schroeter-Brauss, Wecker \& Henrici 2018: 145).

Dabei könne beispielsweise Arbeitsanweisungen und Handlungsschritte, wie etwa in Bezug auf die Operatoren, visualisiert werden. Ein Beispiel dazu finden Sie unter http://euliteracy.eu/wp-content/uploads/2018/10/MLM_Operatoren_im_Unterricht.pdf [16.12.2020]. Auf dieser Ebene können aber auch verschiedene Methoden eingebunden werden. Die Schüler*innen können etwa Wortfelder kreieren. Dazu erhalten die Schüler*innen von der Lehrperson eine Menge an Fachbegriffen und Satzteilen in ungeordneter Reihung als Sprachmaterial. In dieser Menge können sich Begriffspaare verstecken oder es können Sätze aus den Bruchstücken gebildet werden. Zusätzlich können auch „Wortgeländer“ zu bestimmten Prozessen notiert werden. Dabei werden grundlegende Wortelemente zur Beschreibung eines Prozesses verschriftlicht. Dies kann den Erwerb spezifischer Satzstrukturen und deren Verbalisierung unterstützen (vgl. Hepp, Krüger \& Leisen 2003/2005: 4).

\section{Textebene:}

Auf Textebene steht einerseits die Auseinandersetzung mit fachtypischen Textmustern, sowie deren Produktion im Vordergrund (vgl. Morawski \& Budke 2017: 31; vgl. Budke \& Kuckuck 2018: 24f.). Andererseits soll hier im Zuge der sprachlichen Werkzeugkiste vor allem die Reflexion des eigenen sprachlichen Wissens und Könnens adressiert werden. In diesem Zusammenhang kann die Methode der „Verstehensinseln“ herangezogen werden. Dabei notieren Schüler*innen die Aspekte eines Themas, die sie bereits verstanden haben, auf ihrer Insel (vgl. Leisen 2015:15). Sie haben sie sozusagen schon ins Trockene gebracht. Die Dinge, bei denen sie sich noch unsicher fühlen, können abseits der Insel, im Wasser, vermerkt werden. Dies kann aber auch abseits der Methode mittels eines „Was ich gelernt habe“- Satzes oder genau umgekehrt durch einen „Hier fühle ich mich noch unsicher“ - Satz erfolgen. Darüber hinaus können aber auch Visualisierungstechniken, wie Mindmaps oder Begriffsnetze, eingesetzt werden, um inhaltliche Zusammenhänge der Texte zu veranschaulichen (vgl. Hepp, Krüger \& Leisen 2003/2005: 3). Für eine vernetzte Auseinandersetzung mit den inhaltlichen Themen ist in der sprachlichen Werkzeugkiste aber auch Platz. Hier bietet es sich an Texte bzw. Berichte aus anderen Medien, wie Zeitungen oder sozialen Netzwerken, aber auch Werbungen zum Thema, zu sammeln.

\section{Reflexion:}

Die Schüler*innen sollten den Gebrauch der einzelnen Methoden reflektieren. Die Reflexion kann dabei auch kreativ erfolgen: Es können Dialoge, Gedichte, Tagebucheinträge oder eigene Werbeslogans verfasst bzw. gestaltet werden. Dadurch setzen sich die Schüler*innen aktiv und reflexiv mit ihrer Portfolioarbeit auseinander. Wichtig ist natürlich dabei auch ein kontinuierliches Feedback seitens der Lehrperson.

Beim Befüllen der sprachlichen Werkzeugkiste sind der Kreativität der Schüler*innen, wie auch der der Lehrperson, keine Grenzen gesetzt. Wie oft und in welchem Rahmen sie eingesetzt wird, steht allen Beteiligten offen. Allerdings ist ein regelmäßiger Einsatz zu empfehlen, um ein möglichst großes Methodenrepertoire entstehen zu lassen und die Schüler*innen nachhaltig sprachlich zu fördern. Viel Spaß bei der Umsetzung!

\section{Literatur:}

Budke, A. \& M. Kuckuck (2017): Sprache im Geographieunterricht. In: Budke, A. \& M. Kuckuck (Hrsg.): Sprache im Geographieunterricht: Bilinguale und sprachsensible Materialien und Methoden. Waxmann, Münster. S. 7-35.

Hepp, R., A. Krüger \& J. Leisen (2003/2005): Steckbrief Methoden-Werkzeuge. Friedrich, Seelze.

Leisen, J. (2015): Leseverstehen und Leseförderung als Aufgabe aller Fächer. In: Pädagogik.Leben 1. S. 14-15.

http://www.josefleisen.de/downloads/lesen/10\%20Leseverstehen $\% 20$ als $\% 20$ Aufgabe $\% 20$ aller $\% 20 \mathrm{~F} \% \mathrm{C} 3 \%$ A4cher $\% 20 \mathrm{P} \% \mathrm{C} 3 \%$ A4dagogik-leben $\% 202015$.pdf (24.02.2021)

Morawski, M. \& A. Budke (2017): Sprachförderkonzepte in bilingualen Geographieschulbüchern. In: GW Unterricht 148. S. 28-42. DOI: https://doi.org/10.1553/gw-unterrichı Schroeter-Brauss, S., V. Wecker \& L. Henrici (2018): Sprache im naturwissenschaftlichen Unterricht: Eine Einführung. Waxmann, Münster. 


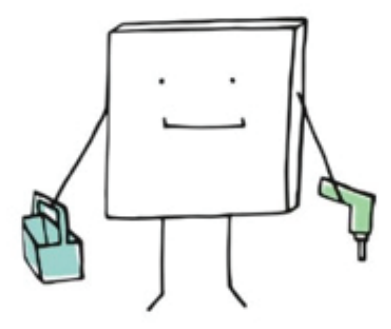

\section{M9 Handout Methoden (für Schüler*innen) \\ Meine \\ Sprachwerkzeuge \\ für den (GW-)Unterricht}

Manchmal ist Sprache sehr schwierig. Methoden sind eine Art Werkzeug, die mir dabei helfen können. In dieser Mappe sammle ich alle meine Werkzeuge, die mir geholfen haben. Wenn ich das oft mache, habe ich viele verschiedene Methoden in meiner „Werkzeugkiste“. Die unterschiedlichen „Werkzeuge“ helfen bei schwierigen Wörtern, Sätzen und Texten. Ich soll aber auch darüber nachdenken, ob mir diese Werkzeuge geholfen haben. Für diese Gedanken ist hier auch Platz.

Wir stellen dir nun einige Werkzeuge für schwierige Wörter, Sätze und Texte vor. Probiere sie aus und überlege, ob und wie sie dir (nicht) geholfen haben. Halte diese Gedanken schriftlich fest.

\section{Werkzeuge für schwierige Wörter:}

Im Unterricht wirst du oft schwierige Wörter kennenlernen. Zu diesen schwierigen Wörtern zählen zum Beispiel Fachbegriffe. Das sind Wörter, die in einem Unterrichtsfach wie GW häufig verwendet werden (zum Beispiel „Globalisierung" und „Äquator"). Damit du dir diese Fachbegriffe gut merkst, kannst du eine eigene Definition des Wortes schreiben. Eine Definition ist eine genaue Beschreibung des Wortes. Du kannst aber auch ein passendes Bild zu diesem Fachbegriff kleben oder dazu malen. Nach einem abgeschlossenen Thema kannst du auch überlegen, welches Wort häufig im Unterricht gebraucht wurde. Dieses Wort kannst du dann als „Wort der Woche“ in deiner sprachlichen Werkzeugkiste sammeln.

Ein weiteres Werkzeug für schwierige Wörter ist das „Themen-ABC“. Beim „Themen-ABC“ versuchst du alle Fachbegriffe, die dir zu einem bestimmten Thema einfallen, zu sammeln. Dabei ist wichtig, dass du sie dem Alphabet nach ordnest.

\section{Werkzeuge für schwierige Sätze:}

Im Unterricht musst du oft sehr lange und schwierige Sätze lesen. Um dir dabei zu helfen, gibt es verschiedene Werkzeuge für schwierige Sätze: In deinem Schulbuch findest du viele verschiedene Aufgaben. Vielleicht fällt dir auf, dass diese Aufgaben immer mit bestimmten Wörtern anfangen. Diese Wörter nennt man „Operatoren“. Die Operatoren erklären uns, was wir bei der Aufgabe machen müssen. Zu den Operatoren gehören Wörter wie „nenne“, „,beschreibe“, „,erkläre“ und noch viele andere Wörter. Damit du dir merkst, was diese Operatoren bedeuten, kannst du dir eine Zeichnung dazu machen. Wir geben dir dazu ein Beispiel: Beginnt eine Aufgabe mit „Bewerte“, dann sollst du Dinge aufschreiben, die du gut oder schlecht findest. Zum Operator „bewerte“ könntest du zum Beispiel so eine Zeichnung machen:

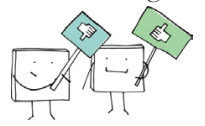

Eine weitere Methode für schwierige Sätze sind die „Wortfelder“. Um diese Methoden anwenden zu können, bekommst du von deiner Lehrerin oder deinem Lehrer Material. In diesem Material befinden sich verschiedene Wortpaare oder Satzteile, die du ordnen musst.

Du kannst aber auch die Methode „Wortgeländer“ verwenden. Diese hilft dir besonders bei bestimmten Abläufen (Prozessen - zum Beispiel wie Menschen miteinander handeln), die du im Unterricht kennenlernst. Wortgeländer sind bestimmte Wörter und Sätze, die dir beim Beschreiben eines Prozesses helfen können.

\section{Werkzeuge für schwierige Texte:}

Im Unterricht gibt es aber nicht nur schwierige Wörter und Sätze, auch die Texte sind oft schwer zu verstehen. Um dir dabei zu helfen, gibt es auch Werkzeuge für schwierige Texte:

Eine dieser Methoden ist die „Verstehensinsel“. Du liest zuerst einen Text. Nach dem Lesen überlegst du, was du bereits verstanden hast. Du malst dann in die Mitte eines Blatt Papiers einen Kreis. Dieser Kreis stellt deine Verstehensinsel dar. Die Dinge, die du verstanden hast, schreibst du auf deine Verstehensinsel. Die Dinge, bei denen du dir noch unsicher bist, kannst du ins Wasser rund um deine Insel schreiben. Danach liest du den Text nochmal. Du überlegst, ob sich einige Dinge aus dem Wasser nun bereits schon auf der Insel im Trockenen befinden.

Du kannst auch nach dem Lesen eines Textes einen Satz aufschreiben, der beschreibt, was du beim Lesen gelernt hast. Du kannst aber auch aufschreiben, wo du dich noch unsicher fühlst und was du noch nicht ganz verstanden hast.

Bei schwierigen Texten kann es dir aber auch helfen, den Inhalt bildlich darzustellen. Dazu kannst du Methoden wie „Mindmaps“ oder „Begriffsnetze“ verwenden. Durch diese Methode bekommst du einen Überblick über den Inhalt des Textes.

Du kannst in deiner sprachlichen Werkzeugkiste aber auch Bilder oder Texte aus der Zeitung, der Werbung oder dem Internet

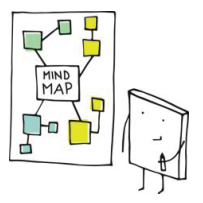
sammeln. Das sollen Bilder oder Texte sein, die zum Unterrichtsthema passen.

\section{Hat dir die Methode geholfen?}

Um etwas zu lernen, ist es wichtig, darüber nachzudenken, ob dir die Methode auch geholfen hat. Mach dir Gedanken darüber und schreibe sie in deine sprachliche Werkzeugkiste. Dabei ist auch Platz für kreative Ideen: Du kannst einen Tagebucheintrag oder ein Gedicht schreiben. Du kannst dir auch einen Dialog, also ein Gespräch, mit der Methode ausdenken, der beschreibt, ob sie dir geholfen hat.

Welche Methode du ausprobieren möchtest, kannst du selbst entscheiden. Es ist jedoch wichtig, regelmäßig zu üben. Dadurch sammelst du viele sprachliche Werkzeuge für deine Werkzeugkiste. 